\title{
Editorial
}

\section{Stochastic Systems 2013}

\author{
Weihai Zhang, ${ }^{1}$ Xuejun Xie, ${ }^{2}$ Suiyang Khoo, ${ }^{3}$ Guangchen Wang, ${ }^{4}$ and Wuquan $\mathrm{Li}^{5}$ \\ ${ }^{1}$ College of Information and Electrical Engineering, Shandong University of Science and Technology, Qingdao 266590, China \\ ${ }^{2}$ Institute of Automation, Qufu Normal University, Qufu 273165, China \\ ${ }^{3}$ School of Engineering, Deakin University, Geelong, VIC 3216, Australia \\ ${ }^{4}$ School of Control Science and Engineering, Shandong University, Jinan 250061, China \\ ${ }^{5}$ School of Mathematics and Information, Ludong University, Yantai 264025, China \\ Correspondence should be addressed to Weihai Zhang; w_hzhang@163.com
}

Received 6 August 2013; Accepted 6 August 2013

Copyright (C) 2013 Weihai Zhang et al. This is an open access article distributed under the Creative Commons Attribution License, which permits unrestricted use, distribution, and reproduction in any medium, provided the original work is properly cited.

Randomness is inherent in reality, but it is often ignored due to the resulting difficulties. Stochastic control plays a central and significant role in modern control theory, which presents a valid tool for dealing with the randomness in the forms of Brownian motion and white noise. With the fast development of biology systems, mathematical finance, insurance, real estate, multiagent, and network control, a lot of new, challenging stochastic-control problems are springing up, which covers the fields of leader-follower game, stochastic filtering, stability, and so forth. These problems are desired to be deeply investigated by using more advanced theories and tools. To reflect the most recent advances in these fields, we are determined to organize this special issue.

This special issue focuses on stochastic-control systems governed by Ito-type stochastic differential equations and discrete-time stochastic difference equations together with their applications to control, filtering, communication, manufacturing, and fault detection. Topics covered in this issue include (i) stochastic modeling, stability, and stabilization analysis, (ii) stochastic robust/optimal/near-opti$\mathrm{mal} /$ adaptive control, (iii) stochastic filtering and estimation, (iv) stochastic differential game, (v) small-gain approach to control of stochastic nonlinear systems, (vi) nonlinear risk measure including g-expectation, (vii) applications of stochastic-control theory to finance, economics, insurance, real estate, manufacturing systems, fault detection, and networked control systems. This special issue has received a total 51 of submitted papers with only 31 papers accepted.
There are 9 manuscripts on the subject "stochastic modeling, stability, and stabilization analysis." In the following, we give a brief summary. The paper entitled "Riskbased predictive maintenance for safety-critical systems by using probabilistic inference" by $\mathrm{T}$. Xu et al. proposes a two-slice temporal Bayes net and risk-based maintenance model for safety critical systems. A new queuing model is proposed by V. Sağlam and M. Zobu in the paper " $A$ two-stage model queueing with no waiting line," where the new Markovian model consists of two consecutive channels and no waiting line between channels. In "Probabilistic value-centric optimization design for fractionated spacecrafts based on unscented transformation," M. Xu et al. quantitatively assess the impacts of various fractionated spacecraft architecture strategies on the lifecycle cost, mass, propellant usage, and mission lifetime of pointing- intensive, remote sensing mission spacecraft. Two Markov chain models have been presented to simulate pitting corrosion in "Markov chain models for the stochastic modeling of pitting corrosion" by A. Valor et al. In "Exponential stability of stochastic nonlinear dynamical price system with delay," W. Zhu et al. give some sufficient conditions of exponential stability, and corollaries for such price systems are established by virtue of Lyapunov function. In "Stochastic stability for time-delay Markovian jump systems with sector-bounded nonlinearities and more general transition probabilities" by D. Ye et al., a more general transition probability matrix is considered, and the delay-dependent sufficient conditions for stability are 
derived in terms of linear matrix inequalities. By constructing a novel piecewise Lyapunov-Krasovskii functional candidate, discrete-time stochastic exponential stability is studied in "Exponential stability results of discrete-time stochastic neural networks with time-varying delays" by Y. Li. A. Gu obtains the synchronization between two solutions and among different components of solutions under certain dissipative conditions in the manuscript "Synchronization of coupled stochastic systems driven by $\alpha$-stable Levy noises." "Robust passivity and feedback design for nonlinear stochastic systems with structural uncertainty" by Z. Lin et al. discusses the robust passivity and global stabilization problems for a class of uncertain nonlinear stochastic systems with structural uncertainties.

Closely related to controlled stochastic differential equations are 3 contributions. J. Shi and Z. Yu's paper "Relationship between maximum principle and dynamic programming for stochastic recursive optimal control problems and applications" establishes a relationship between maximum principle and dynamic programming. A maximum principle and a verification theorem for optimal control are presented in "Optimality conditions for optimal control of jump-diffusion SDEs with correlated observations noises" by $\mathrm{H}$. Xiao. The third contribution is for the relationship between functional forward-backward stochastic differential equations and path-dependent PDEs. Within the framework of functional Ito calculus, S. Ji and S. Yang introduce a path-dependent PDE and prove that its solution is uniquely determined by a functional forwardbackward stochastic differential equation.

There are 5 papers concerned about stochastic filtering and estimation. J. Liu et al. propose an improved particle filter for multiple maneuvering target tracking in the paper " $\mathrm{Mul}$ tiple maneuvering target tracking by improved particle filter based on multi-scan JPDA." "Maximum likelihood estimation of the VAR (1) model parameters with missing observations" by H. Mouriño and M. I. Barão is about maximum likelihood estimation based on monotone missing data pattern. " $H_{\infty}$ estimates for discrete-time Markovian jump linear systems" by M. H. Terra et al. deals with the $H_{\infty}$ filtering problem for discrete-time Markovian jump linear systems based on the game theory. Based on the matrix inequality technique, the $H_{\infty}$ filtering problem is studied by Z. Yan and Y. Huang in their paper "Robust $H_{\infty}$ filter design for Itô stochastic pantograph systems." In the paper "Optimal fusion filtering in multisensor stochastic systems with missing measurements and correlated noises" by R. Caballero-Águila et al., the optimal least-square linear estimation problem is addressed for a class of discrete-time multisensor linear stochastic systems.

There are also 3 contributions on stochastic differential games. Using the theory of backward stochastic differential equations, L. Wei and Z. Wu study stochastic recursive zerosum differential game and mixed zero-sum differential game problem. S. Ji et al. use a dynamic programming method to investigate stochastic differential games derived by functional forward-backward stochastic differential equations in "The dynamic programming method of stochastic differential game for functional forward-backward stochastic system." "Governance mechanism for global greenhouse gas emissions: a stochastic differential game approach" by W. Yu and B. Xin proposes cooperative and noncooperative stochastic differential game models to describe greenhouse gas emissions decision makings of developed and developing countries and calculates their feedback Nash equilibrium and the Pareto optimal solution and characterizes parameter spaces.

The subject on financial engineering and insurance has occupied 6 contributions. These contributions include, for example, convertible bond pricing, option pricing, optimal dividend strategy, and optimal portfolio selection. X. Guo and $\mathrm{H}$. Wang study a pricing problem for convertible bond via reflected backward stochastic differential equations. X. Ruan et al. consider option pricing with risk-minimization criterion in an incomplete market with a finite difference method. Y. Fang and Z. Qu investigate a risk model including a constant force of interest and obtain an optimal strategy. W. Yu focuses on a discrete insurance risk model with stochastic premium income. Z. Huang and D. Zhang propose a model of corporate optimal investment with consideration of the influence of inflation and the difference between the market opening and market closure. J. A. Londoño considers a statedependent utility problem in an incomplete market and develops a theory of markets when the processes are the generalization of Brownian flows on manifolds.

Other applications of stochastic-control theory can be found in the following: M. Zobu and V. Sağlam investigate the control of traffic intensity based on SPRT method. One paper entitled "Fault detection for linear discrete time-varying systems with measurement packet dropping" by Y. Li et al. introduces an adjoint operator-based optimization method; the analytical optimal solution is derived via solving a modified Riccati equation. In the paper " $A$ novel detection scheme for EBPSK system," X. Chen and L. Wu introduce a novel solution for EBPSK communication systems, and a joint detection algorithm is given to eliminate the ISI coming from the SIF. Y. Fang et al. establish a model of the travel time of right-turning vehicles on secondary street at unsignalized intersections in congested urban traffic condition. X. Yin et al. discuss the discrete memoryless broadcast channels (DMBCs) with noiseless feedback; the entire capacity-equivocation regions of two models of the DMBCs with noiseless feedback are obtained.

\section{Acknowledgments}

This special issue represents an exciting and insightful snapshot of the current stochastic system research. As the Lead Guest Editor of this special issue, I would like to express my sincere gratitude to my four coeditors for helping me to undertake this project with a wonderful accomplishment. We are also deeply appreciative of interesting authors who have submitted their papers to the special issue.

$$
\begin{array}{r}
\text { Weihai Zhang } \\
\text { Xuejun Xie } \\
\text { Suiyang Khoo } \\
\text { Guangchen Wang } \\
\text { Wuquan Li }
\end{array}
$$




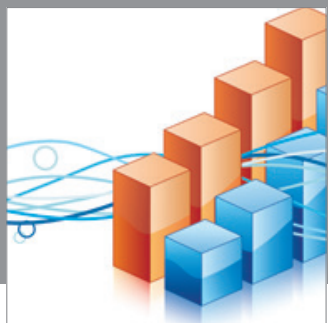

Advances in

Operations Research

mansans

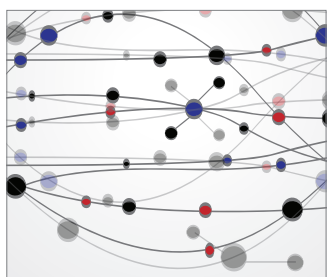

The Scientific World Journal
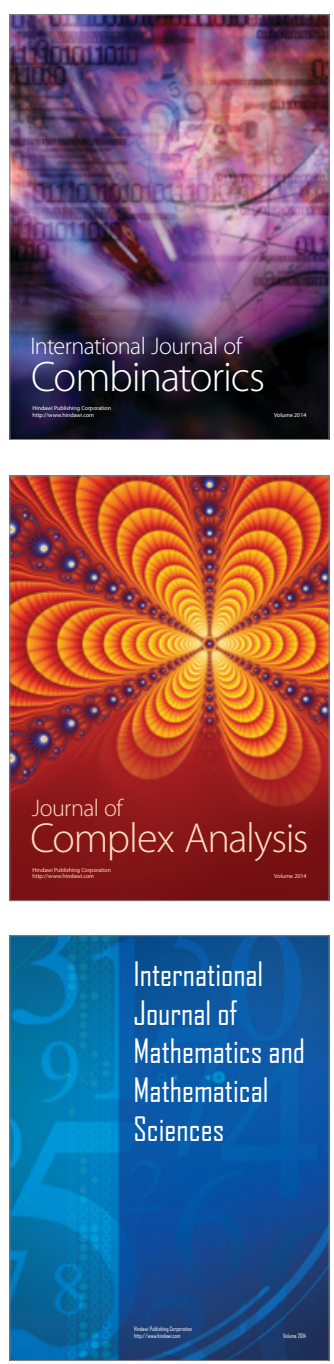
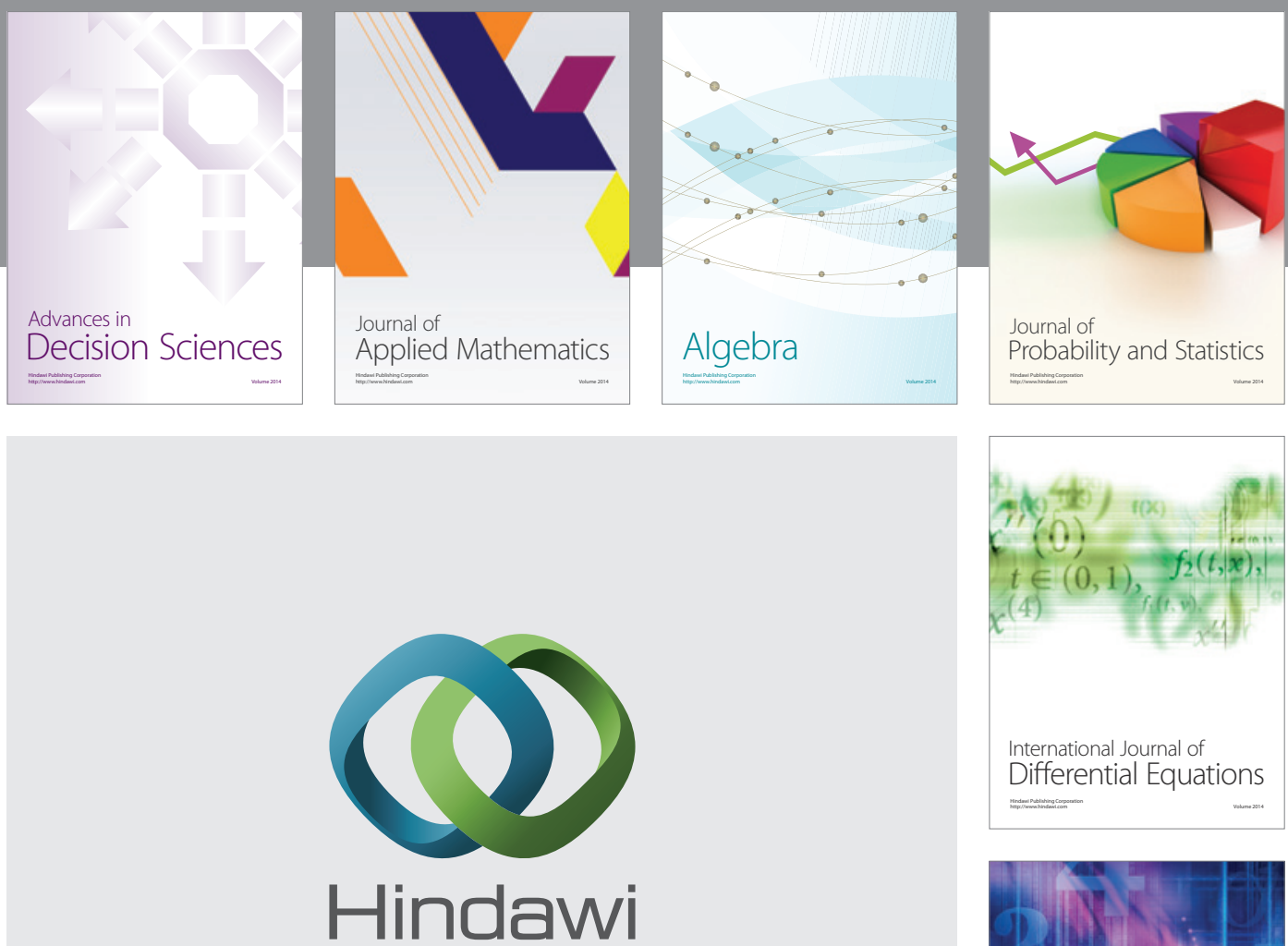

Submit your manuscripts at http://www.hindawi.com
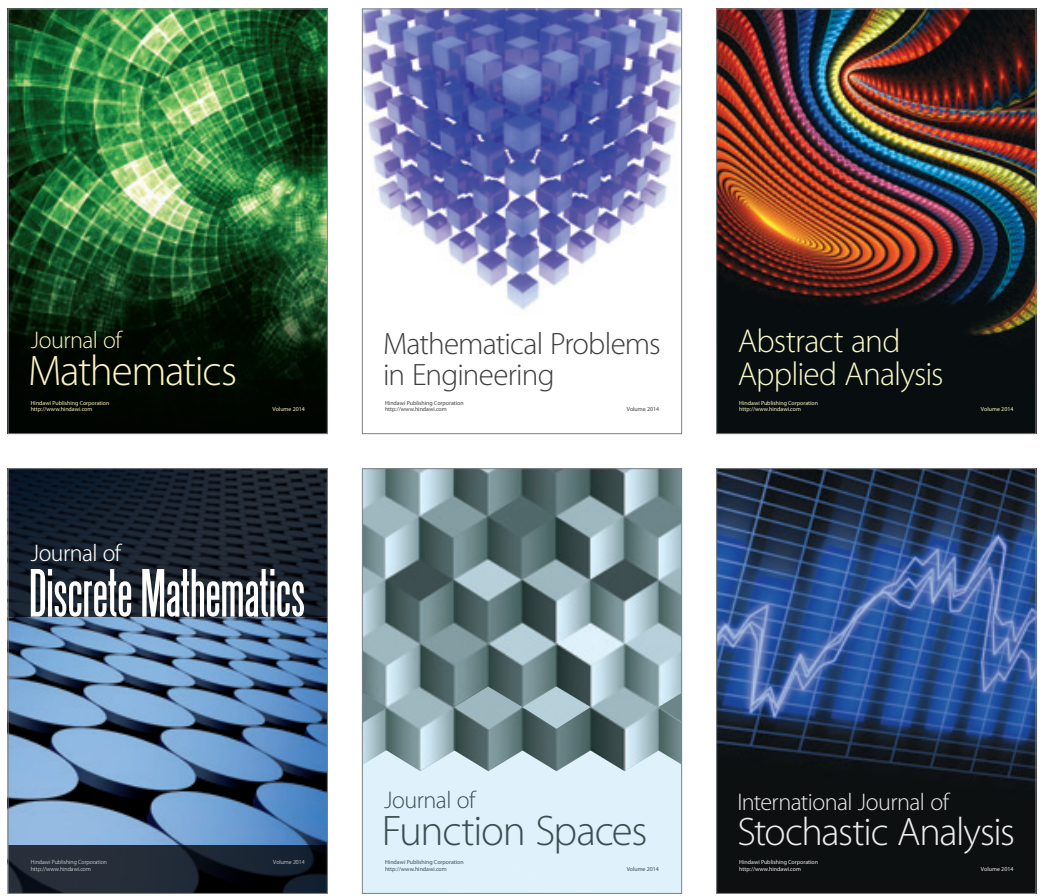

Journal of

Function Spaces

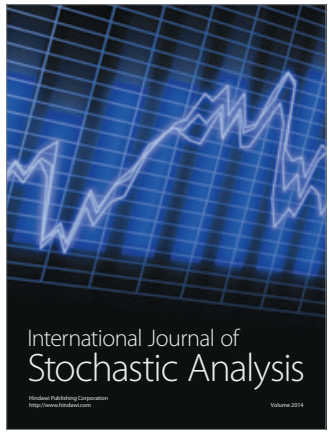

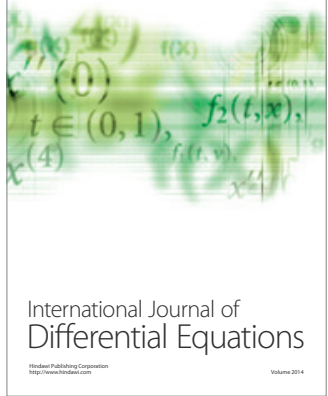
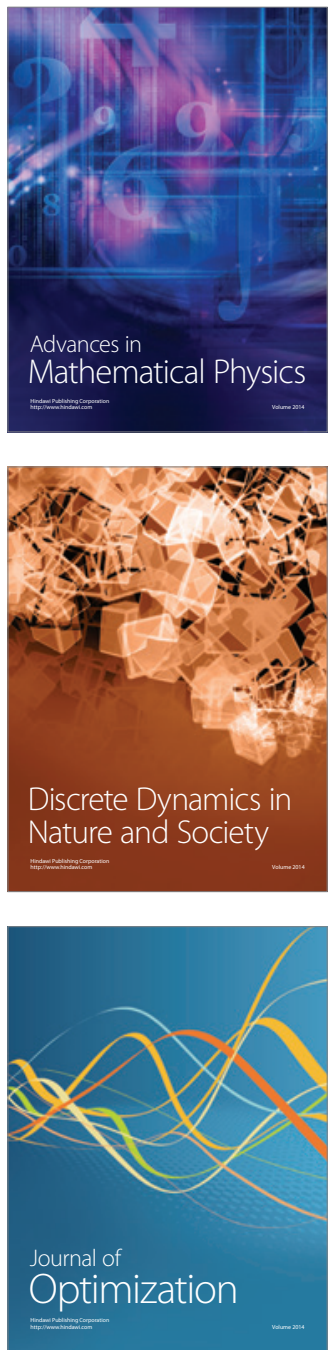